\title{
Active capture and stabilization of temporal solitons in microresonators
}

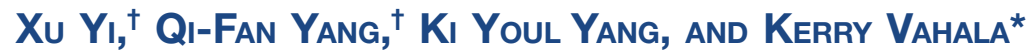 \\ T. J. Watson Laboratory of Applied Physics, California Institute of Technology, Pasadena, California 91125, USA \\ *Corresponding author: vahala@caltech.edu
}

Received 10 March 2016; revised 1 April 2016; accepted 1 April 2016; posted 4 April 2016 (Doc. ID 260853); published 26 April 2016

\begin{abstract}
Soliton mode locking and femtosecond pulse generation have recently been demonstrated in high- $Q$ optical microcavities and provide a new way to miniaturize frequency comb systems, as well as create integrated comb systems on a chip. However, triggering the mode-locking process is complicated by a well-known thermal hysteresis that can destabilize the solitons. Moreover, on a longer time scale, thermal drifting of the cavity resonant frequency relative to the pumping frequency causes loss of mode locking. In this Letter, an active feedback method is used both to capture specific soliton states and to stabilize the states indefinitely. The capture and stabilization method provides a reliable way to overcome thermal effects during soliton formation and to excite a desired number of circulating cavity solitons. It is also used to demonstrate a low pumping power of $22 \mathrm{~mW}$ for generation of microwave-repetition-rate solitons on a chip. () 2016 Optical Society of America
\end{abstract}

OCIS codes: (190.0190) Nonlinear optics; (140.3945) Microcavities; (190.3270) Kerr effect; (190.4970) Parametric oscillators and amplifiers; (190.7110) Ultrafast nonlinear optics.

http://dx.doi.org/10.1364/OL.41.002037

Thermal effects complicate the operation of nano- and microscale systems. Power buildup in their small volumes can result in desirable system features being masked or inhibited by parasitic heating. High- $Q$ optical microcavity systems provide a well-known example. Here, heating accompanies resonant power buildup of a coupled optical field $[1,2]$. The heating shifts the resonant frequency through the temperature dependence of the refractive index. As a result, power coupling into the resonant mode features a hysteresis such that pump frequencies slightly blue detuned relative to the resonance are stable (the socalled thermal-locking regime [2]), while red-detuned frequencies are unstable. Even though many high- $Q$ microcavity applications can operate with a blue-detuned pump or a pump that is locked to cavity line center, red-detuned operation of the pump relative to the microcavity line center is essential for soliton mode locking [3-5].

We have recently shown that a single-soliton state can be stabilized indefinitely by feedback control to the pump laser frequency such that the soliton power is held at a fixed set point [5]. Here, this method is extended to actively capture a specific single- or multi-soliton state, and it is used to demonstrate low power operation of a soliton comb. This shows that the method not only maintains long-term stability of the solitons, but also can dynamically compensate for short-term transients. In addition, important details on the method are presented.

Femtosecond pulse generation through formation of dissipative Kerr cavity solitons has been observed in crystalline [3], silicon nitride [4,6], and silica systems [5]. Solitons are formed when an optical pump is tuned from the blue side of a cavity resonance to the red side. Blue-detuned operation is thermally stable so that parametric oscillation $[7,8]$ and cascaded four-wave mixing (FWM) comb formation [9-20] are readily achieved when the cavity dispersion is anomalous. The FWM process is required to seed the solitons. With continued pump tuning to the red side of the cavity resonance, their formation leads to abrupt, "step-like" features in the intracavity power [see Fig. 1(a)] [3] and an accompanying cavity tuning transient. Curiously, while the red-detuned regime is normally unstable, once formed, solitons stabilize this regime. This happens because the average circulating soliton power increases with further red-detuning of the pump laser (i.e., opposite to the normal continuous-wave circulating power detuning dependence). This unusual detuning dependence of soliton power thereby enables red-detuned operation, provided that the soliton state persists long enough so as to permit decay of the initial nonsoliton (destabilizing) transient.

Two passive methods have been demonstrated to overcome thermal destabilization during soliton formation. In magnesium fluoride resonators, where the thermal effect is relatively weak, the red-detuned soliton regime can be achieved by pump laser sweep rate control [3]. In cases where there is a stronger thermal effect, the two-step "power kicking" protocol was developed in silicon nitride resonators [4] and has also been applied in silica resonators [5]. However, these passive approaches are challenging to implement, requiring a customized pump power time dependence. In addition, excitation of a specific soliton state is not guaranteed and, significantly, the soliton power and pulse width still vary as a result of long-term drift of the cavity and pump laser frequencies. Ultimately, these drifts result in loss of the soliton state when the cavity-pump detuning drifts to outside the so-called soliton existence detuning range $[3,5,21,22]$. Long-term stabilization of solitons has been proposed by using frequency-swept phase modulation 


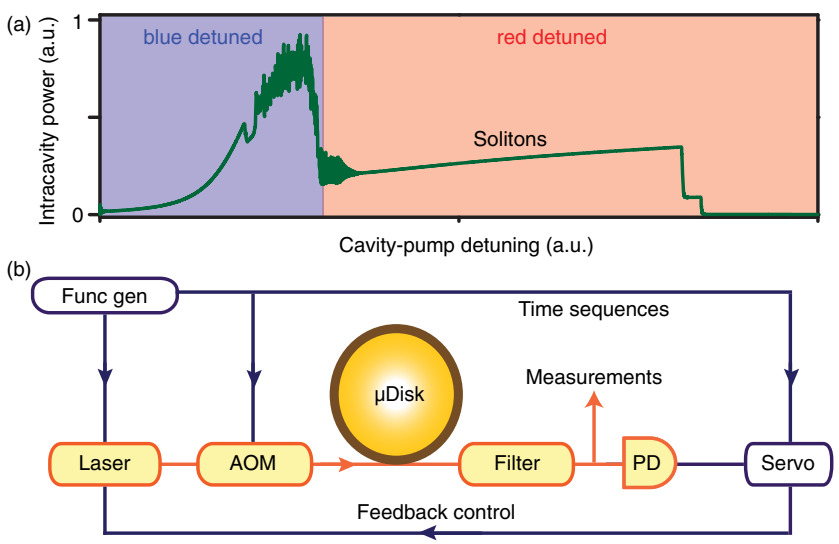

(c)

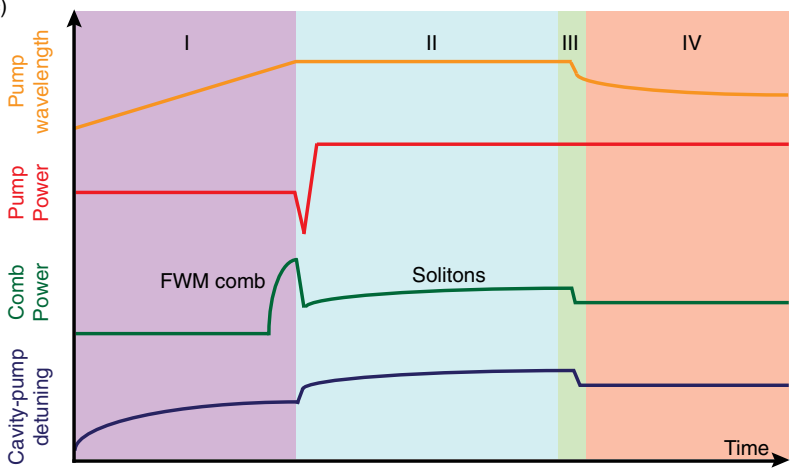

Fig. 1. (a) Simulated intracavity power in which the pump laser scans over the resonance from the blue side to the red side. The steps on the red-detuned side indicate soliton formation. (b) Schematic of experimental setup. (c) Four phases of feedback-controlled soliton excitation: (I) pump laser scans into cavity resonance from the blue-detuned side; (II) laser scan stops and pump power is reduced $(\sim 10 \mu \mathrm{s})$ to trigger solitons, and then increased $(\sim 100 \mu \mathrm{s})$ to extend soliton existence range; (III) servo control is engaged to actively lock the soliton power by feedback control of laser frequency; (IV) lock sustains and solitons are fully stabilized. The cavity-pump detuning (vertical axis) is relative to the hot cavity resonant frequency.

of a pump laser; however, this method has not been demonstrated [23]. Concerning generation of specific numbers of solitons, injection of "addressing" pulses has been used in fiber soliton lasers to trigger specific numbers of solitons [24], and laser backward tuning has been recently reported in microresonators [23]. However, neither technique achieves stabilization. In addition, a separate mode locked laser is required to generate the "addressing" pulses.

The capture and stabilization method described here is simple to implement, relying on servo control of the pump laser frequency by measuring the soliton average power. The key parameter in understanding this servo control method is the relative cavity-pump detuning, which, for fixed cavity loading, determines soliton power and pulse width. The one-to-one relationship between detuning and several soliton properties was recently noted to provide a way to provide long-term locking of the laser cavity detuning [5]. Specifically, the soliton power is given by the following expression $[3,5,21,22]$ :

$$
P_{\text {sol }}=\frac{2 \eta A_{\mathrm{eff}}}{n_{2} Q} \sqrt{-2 n c \beta_{2} \delta \omega},
$$

where $c$ is light speed, $A_{\text {eff }}$ is the effective mode area, $n_{2}(n)$ is the nonlinear index (refractive index), $Q$ is the loaded quality factor, $\eta=Q / Q_{\text {external }}$ is the coupling coefficient, $\beta_{2}$ is the group velocity dispersion, and $\delta \omega=\omega_{\text {cavity }}-\omega_{\text {laser }}$ is the cavity-pump frequency detuning ( $\omega_{\text {cavity }}$ is temperature dependent). It is important to note that the typical frequency detuning values for soliton generation are in the range of $10 \mathrm{~s}$ of cavity linewidths. As a result, it is not clear how conventional locking methods can be applied in these circumstances. On the other hand, leveraging the one-to-one dependence of soliton power on detuning given by Eq. (1) avoids the complexities associated with actual measurement of the detuning frequency.

The experimental setup in Fig. 1(b) shows the resonator (a high- $Q$ silica resonator described in Refs. [5,25]) pumped with a continuous-wave fiber laser amplified by an erbiumdoped fiber amplifier. The high- $Q$ resonators are $3 \mathrm{~mm}$ in diameter and produce solitons at a repetition frequency of approximately $22 \mathrm{GHz}$. The laser's frequency is piezo controlled by a function generator and the servo box. An acoustic-optic modulator (AOM) is used to control the pump power coupled into the resonator. At the resonator output port, the comb power is separated from the pump power with a fiber Bragg grating filter and is sent to a photodiode (PD). An error signal is generated from the detected photocurrent after subtracting a set point in the servo control box.

The function generator produces signals to create a time sequence for all instruments. The time sequence is composed of four phases illustrated in Fig. 1(c). The first two are similar to the "power kicking" technique [4], with the exception that the precise shape of the pump power waveform is less important than when power kicking is applied alone. In phase I, the laser is first scanned into resonance from the blue side, causing FWM comb formation [9]. In phase II, the laser stops scanning, and the pump power is decreased over a few microseconds by controlling the acousto-optic modulator. This reduces the intracavity power so that the cavity resonance blueshifts due to the Kerr and thermal effects. At some point, the laser wavelength will reside on the red side of the cavity resonance, where soliton formation is possible. The laser power is then ramped to a higher level. Ramp times ranging from $\sim 20 \mu \mathrm{s}$ to $\sim 500 \mu \mathrm{s}$ were used. Ramping the power extends the existence detuning range of the solitons given by $\delta \omega_{\max }=\pi^{2} P_{\text {in }} / 16 P_{\text {th }}[3,5,22]$, where $P_{\text {in }}$ and $P_{\text {th }}$ are the pump power and parametric oscillation threshold [5]. If the waveform used to control the pump ramp is carefully designed, then the soliton can be stabilized during this phase for extended periods. More typically, however, the soliton will be lost on account of the residual thermal transients and the associated cavity frequency drift (i.e., cavitypump detuning frequency drifts beyond the soliton existence range).

To overcome these transients, the servo control is activated in phase III using the function generator. The servo controls the laser frequency to maintain a setpoint for average soliton power. Significantly, the cavity-pump detuning is also locked because it determines the soliton power [see Eq. (1)]. This frequency locking compensates for the short-term thermal transients that normally complicate soliton formation, as well as the long-term thermal drift of the cavity and the pump laser. The net effect is that the soliton can be reliably captured and sustained indefinitely, as illustrated in phase IV. For successful capture of the soliton state, the soliton-free running time in phase II should be 
longer than the turn-on time of feedback loop. In the present case, it is limited by the speed of laser controller and is around $100 \mu$ s.

The active locking method described here not only stabilizes the soliton power, but also stabilizes all other soliton characteristics that depend on laser cavity detuning. For example, the soliton pulse width, spectral line power, and Raman-induced selffrequency shift are stabilized [5]. In addition, using this locking method, it is possible to optimize the detuning for maximum efficiency [5]. Moreover, while there exists a combination of "power kicking" parameters that enable a soliton to be passively excited, the passive-excitation parameter window is small and, hence, difficult to achieve. On the other hand, active capture is insensitive to the precise excitation window, since it requires only that the soliton appear long enough for servo locking to engage.

The demonstration of active capture and locking of a single soliton is presented in Fig. 2. The comb power is shown in red, and a Mach-Zehnder interferometer (MZI) signal in blue is used to track the pump laser frequency (the free spectral range of the interferometer is $40 \mathrm{MHz}$ ). The MZI is a similar design to that used in [25] and incorporates two, fiber-bidirectional couplers with about a 5 meter path length imbalance. The MZI signal gives an indication of tuning of the pump relative to the interferometer (which is stable over the time period of the scan). Soliton excitation without active capture is shown in Fig. 2(a). Here, the power kicking is employed and the soliton persists for about $20 \mathrm{~ms}$ before destabilizing. The MZI signal shows that the pump laser is thermally drifting during this period. In Fig. 2(b), the feedback loop is switched on around $2 \mathrm{~ms}$ after the power kicking. A zoomed-in view is provided in Fig. 2(c). During phase II, it is interesting to observe the transition from the FWM comb to the soliton step which is accompanied by an abrupt decrease in comb power at $\sim 3.2 \mathrm{~ms}$ (red trace). In addition, in the early part of phase II at $\sim 3.5 \mathrm{~ms}$, a further decrease in comb power indicates thermal drift from

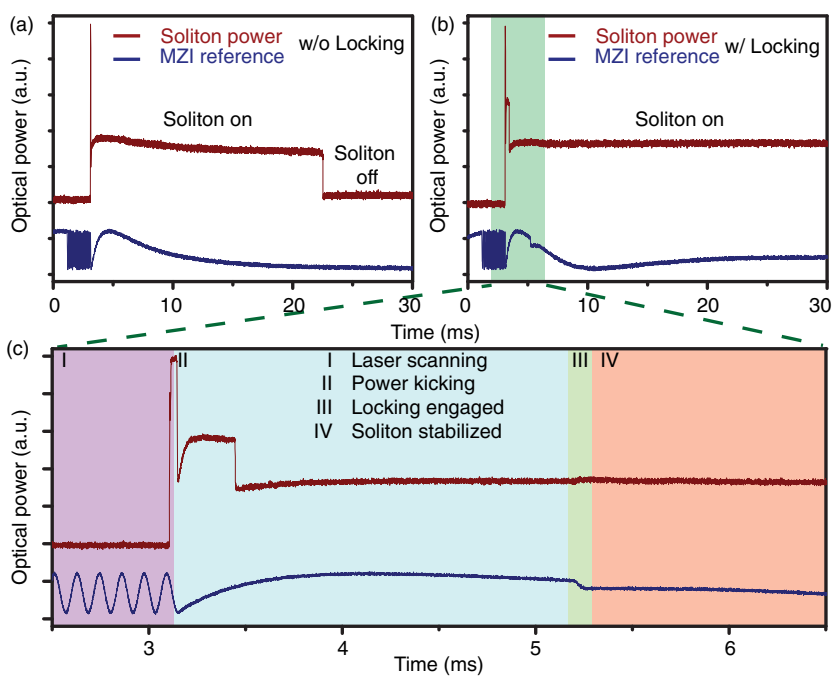

Fig. 2. Demonstration of capture and locking of a soliton state. (a) Soliton excitation with "power kicking," but no active locking is shown. The soliton state destabilizes around $22 \mathrm{~ms}$ due to thermal transients. Soliton power is shown in red, and a MZI reference is in blue. (b) Soliton excitation with active locking is shown with conditions similar to panel (a). (c) Zoom-in view of panel (b). The four phases are indicated using the same background color scheme as in Fig. 1. a two-soliton to a single-soliton state. During phase III, there is a jump in the MZI signal upon engaging the servo control $(\sim 5.2 \mathrm{~ms})$, and stable comb power indicates that the feedback loop is activated. Soliton stability upon locking is confirmed by measuring optical spectra and by studying time-domain intensity autocorrelation. The left panel in Fig. 3(a) shows the locking of a single-soliton state. The corresponding hyperbolic-secant-squared optical spectrum is presented in the left panel of Fig. 3(b), and the single-soliton autocorrelation is confirmed in the left panel of Fig. 3(c). The 46 ps time interval of the two autocorrelation peaks corresponds to the $22 \mathrm{GHz}$ cavity repetition rate.

In addition to the capture and stabilization of single-soliton states, the method described here is able to selectively lock to different soliton states by adjusting the locking set point. For an $N$ soliton state, the average soliton power is $N$ times that of a single-soliton state (assuming the same laser cavity detuning). Therefore, by setting the locking power to roughly $N$ times that of the single-soliton state power it is possible to capture states containing $N$ solitons. The magnitude of the setpoint is mathematically determined by Eq. (1). In the experiment, the desired setpoint is observable by monitoring the soliton power in traces such as Fig. 2(c). For example, a narrow range of setpoint power levels is possible for the single-soliton step in that figure. Each power level corresponds to a distinct cavitypump detuning given by Eq. (1).

In the experiment, a two-soliton state is captured and stabilized. Simulation shows that multi-soliton states have a smaller laser cavity detuning existence range due to perturbation from avoided mode crossings and higher order dispersion [26]. As a result, the two-soliton state observed here is only stable for a few ms under free running conditions. However, by setting

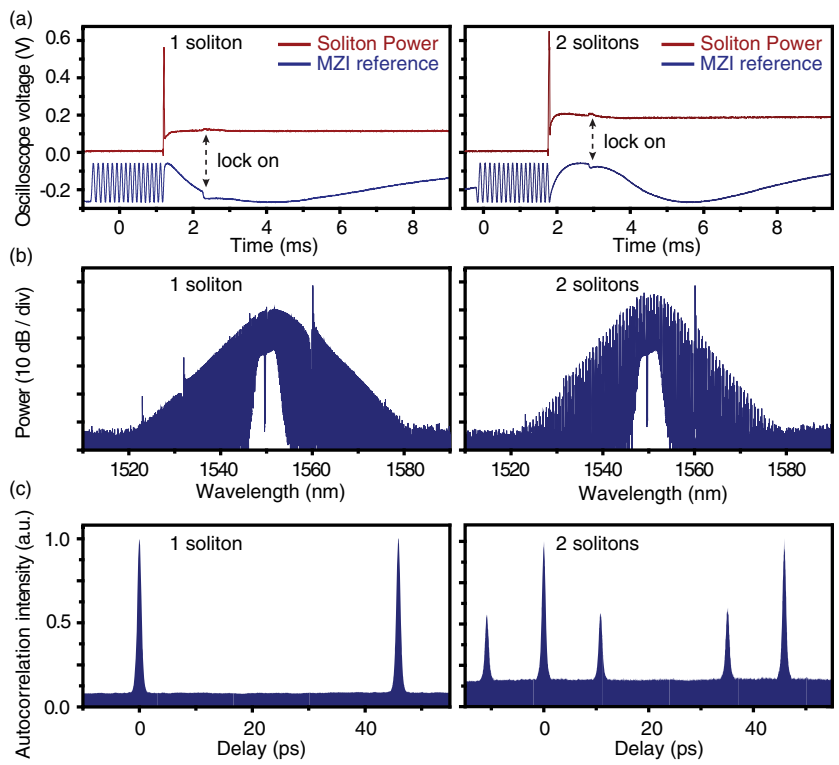

Fig. 3. Controlled excitation of different soliton states. (a) Excitation sequence for single- and two-soliton states. The locking point of the comb power for the two-soliton state is set to twice that of the single-soliton state. Soliton power is shown in red. A MZI reference trace (blue) is used to infer the pump laser frequency. The servo locking point is indicated by an arrow. (b) Optical spectra of the single- and two-soliton states. (c) Intensity autocorrelation of the single- and two-soliton states. 


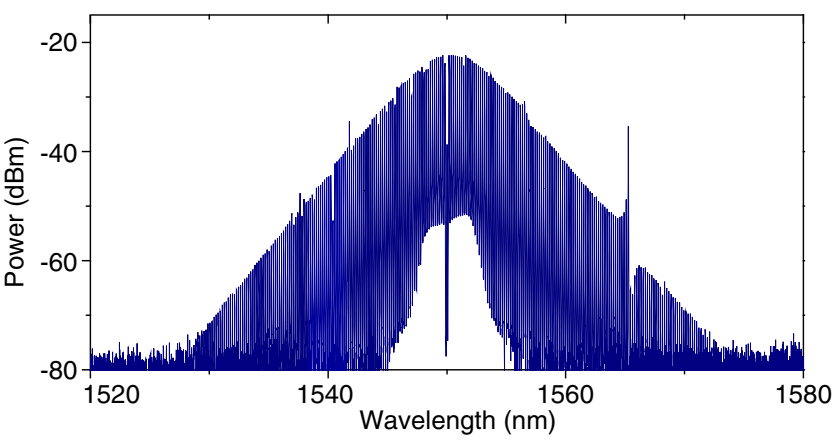

Fig. 4. Single-soliton optical spectrum at a $22 \mathrm{GHz}$ repetition frequency stabilized at a low pumping power of $22 \mathrm{~mW}$.

the servo locking to occur earlier than for the single-soliton state described above and by setting the servo locking level to approximately $2 \times$ the single-soliton level [locking voltage is increased from $0.1 \mathrm{~V}$ to $0.2 \mathrm{~V}$; see right panel in Fig. 3(a)], a two-soliton state is readily captured and stabilized. In the measurement, the locking was set to occur $1 \mathrm{~ms}$ after power kicking. The optical spectrum [Fig. 3(b)] and autocorrelation [Fig. 3(c)] confirm the generation of the two-soliton state.

Because the detuning is locked, it is possible to dramatically reduce the soliton existence range to minimize the pumping power. By reducing the soliton power set point, together with pump power, so as to closely match cavity-pump detuning with soliton existence detuning, a single soliton requiring only $22 \mathrm{~mW}$ of pumping power was achieved (Fig. 4). The number is all the more interesting, as the repetition frequency is at a detectable rate (approximately $22 \mathrm{GHz}$ ) which imposes greater challenges on low power operation.

The active capture technique provides a practical route for stable soliton excitation in any application of soliton mode locking, including frequency combs. Moreover, the ability to control soliton number is useful in several different applications. Singlesoliton states, for example, are preferred for efficient pulse broadening, as they provide highest peak pulse power upon amplification. They also provide a predictable spectral envelope. Multi-soliton states, on the other hand, provide greater average comb power [27]. Active capture of these multi-soliton states is, in principle, no different from generating a single-soliton state. Blue-detuned, FWM frequency combs have been demonstrated in many material systems $[9,10]$, including magnesium fluoride $[15,17]$, calcium fluoride [11], quartz [14], silica [16], silicon [20], silicon nitride [12,13], aluminum nitride [18], and diamond [19]. The method described here for active capture and stabilization of soliton states should work equally well in any of these systems, provided that their cavities are properly optimized with respect to high $Q$-factor, minimal avoided mode crossing and dispersion.

†These authors contributed equally.

Funding. Defense Advanced Research Projects Agency (DARPA) (HR0011-15-C-0055, W911NF-14-1-0284);
National Aeronautics and Space Administration (NASA) (KJV.JPLNASA-1-JPL.1459106); National Science Foundation (NSF) (PHY-1125565).

\section{REFERENCES}

1. V. Ilchenko and M. Gorodetskii, Laser Phys. 2, 1004 (1992).

2. T. Carmon, L. Yang, and K. Vahala, Opt. Express 12, 4742 (2004).

3. T. Herr, V. Brasch, J. Jost, C. Wang, N. Kondratiev, M. Gorodetsky, and T. Kippenberg, Nat. Photonics 8, 145 (2014).

4. V. Brasch, M. Geiselmann, T. Herr, G. Lihachev, M. Pfeiffer, M. Gorodetsky, and T. Kippenberg, Science 351, 357 (2016).

5. X. Yi, Q.-F. Yang, K. Y. Yang, M.-G. Suh, and K. Vahala, Optica 2 1078 (2015).

6. P.-H. Wang, J. A. Jaramillo-Villegas, Y. Xuan, X. Xue, C. Bao, D. E. Leaird, M. Qi, and A. M. Weiner, "Intracavity characterization of microcomb generation in the single-soliton regime," arXiv preprint arXiv:1603.03154 (2016).

7. T. Kippenberg, S. Spillane, and K. Vahala, Phys. Rev. Lett. 93, 083904 (2004).

8. A. A. Savchenkov, A. B. Matsko, D. Strekalov, M. Mohageg, V. S. Ilchenko, and L. Maleki, Phys. Rev. Lett. 93, 243905 (2004).

9. T. J. Kippenberg, R. Holzwarth, and S. Diddams, Science 332, 555 (2011).

10. P. Del'Haye, A. Schliesser, O. Arcizet, T. Wilken, R. Holzwarth, and T. Kippenberg, Nature 450, 1214 (2007).

11. A. A. Savchenkov, A. B. Matsko, V. S. Ilchenko, I. Solomatine, D. Seidel, and L. Maleki, Phys. Rev. Lett. 101, 093902 (2008).

12. J. S. Levy, A. Gondarenko, M. A. Foster, A. C. Turner-Foster, A. L. Gaeta, and M. Lipson, Nat. Photonics 4, 37 (2010).

13. L. Razzari, D. Duchesne, M. Ferrera, R. Morandotti, S. Chu, B. Little, and D. Moss, Nat. Photonics 4, 41 (2010).

14. S. B. Papp and S. A. Diddams, Phys. Rev. A 84, 053833 (2011).

15. W. Liang, A. Savchenkov, A. Matsko, V. Ilchenko, D. Seidel, and L. Maleki, Opt. Lett. 36, 2290 (2011).

16. J. Li, H. Lee, T. Chen, and K. J. Vahala, Phys. Rev. Lett. 109, 233901 (2012).

17. C. Y. Wang, T. Herr, P. Del'Haye, A. Schliesser, J. Hofer, R. Holzwarth, T. Hänsch, N. Picqué, and T. J. Kippenberg, Nat. Commun. 4, 1345 (2013).

18. H. Jung, C. Xiong, K. Y. Fong, X. Zhang, and H. X. Tang, Opt. Lett. 38 , 2810 (2013)

19. B. Hausmann, I. Bulu, V. Venkataraman, P. Deotare, and M. Lončar, Nat. Photonics 8, 369 (2014).

20. A. G. Griffith, R. K. Lau, J. Cardenas, Y. Okawachi, A. Mohanty, R. Fain, Y. H. D. Lee, M. Yu, C. T. Phare, C. B. Poitras, A. L. Gaeta, and M. Lipson, Nat. Commun. 6, 6299 (2015).

21. A. Matsko, A. Savchenkov, W. Liang, V. Ilchenko, D. Seidel, and L. Maleki, Opt. Lett. 36, 2845 (2011).

22. A. B. Matsko and L. Maleki, Opt. Express 21, 28862 (2013).

23. M. Karpov, H. Guo, E. Lucas, A. Kordts, M. H. P. Pfeiffer, G. Lichachev, V. E. Lobanov, M. L. Gorodetsky, and T. J. Kippenberg, "Universal dynamics and controlled switching of dissipative Kerr solitons in optical microresonators," arXiv preprint arXiv:1601.05036 (2016).

24. F. Leo, S. Coen, P. Kockaert, S.-P. Gorza, P. Emplit, and M. Haelterman, Nat. Photonics 4, 471 (2010).

25. H. Lee, T. Chen, J. Li, K. Y. Yang, S. Jeon, O. Painter, and K. J. Vahala, Nat. Photonics 6, 369 (2012).

26. T. Herr, V. Brasch, J. Jost, I. Mirgorodskiy, G. Lihachev, M. Gorodetsky, and T. Kippenberg, Phys. Rev. Lett. 113, 123901 (2014).

27. C. Bao, L. Zhang, A. Matsko, Y. Yan, Z. Zhao, G. Xie, A. M. Agarwal, L. C. Kimerling, J. Michel, L. Maleki, and A. E. Willner, Opt. Lett. 39, 6126 (2014). 